\title{
Experimental Characterization Of a Double Receiver Dynamic Wireless Charging System
}

\author{
Nicola Campagna \\ Department of Engineering \\ University of Palermo \\ Palermo, Italy \\ nicola.campagna@unipa.it
}

Antonino Oscar Di Tommaso

Department of Engineering

University of Palermo

Palermo, Italy

antoninooscar.ditommaso@unipa.it

\author{
Rosario Miceli \\ Department of Engineering \\ University of Palermo \\ Palermo, Italy \\ rosario.miceli@unipa.it \\ Filippo Pellitteri \\ Department of Engineering \\ University of Palermo \\ Palermo, Italy \\ filippo.pellitteri@unipa.it
}

\author{
Vincenzo Castiglia \\ Department of Engineering \\ University of Palermo \\ Palermo, Italy \\ vincenzo.castiglia@unipa.it \\ Fabio Viola \\ Department of Engineering \\ University of Palermo \\ Palermo, Italy \\ fabio.viola@unipa.it
}

\begin{abstract}
The aim of this work is the characterization of a dynamic wireless charging system low power prototype and the validation of a simplified mathematical model of the employed double $D$ coils. The difference between a single receiver and a dual receiver system is also shown, highlighting how the last one can significantly reduce the costs of the charging infrastructure.
\end{abstract}

Keywords-wireless charging, inductive power transfer, dynamic charging.

\section{INTRODUCTION}

The development of the Electrical Vehicles (EVs) can give a strong contribute to the reduction of the global $\mathrm{CO}_{2}$ emissions but it is slower than it should be for a profitable effect. The answer can be found in the lack of charging infrastructures in several countries and in the so-called rangeanxiety developed by consumers. As a matter of fact, EVs should have higher powerful battery packs in order to be competitive with the traditional internal combustion engine vehicles in terms of autonomy but it obviously implies higher costs for the EVs.

Wireless systems based on the Inductive Power Transfer (IPT) can be a suitable solution [1]. They are based on the inductive coupling between two coils, one connected to the main grid, conventionally named as primary side and the other one mounted on the chassis of the EV (secondary side) [2]. In order to maximize the power transfer, a compensation network, typically a bench of capacitors, is connected to each side coil. Different compensation configurations are possible, as shown in [3]. The primary side is connected to the grid through a power converter able to deliver an alternate voltage guaranteeing the resonance between the coil and the compensation net. At the secondary side, a rectified is interposed between the receiver and the load represented by the battery pack [4].

This structure can be employed for both static and dynamic applications. Static wireless charging represents an advantage in terms of facilities and safety for the user thanks to the lack of physical connections and cables [5]. The dynamic one, for which the primary side is composed by more transmitters distributed on the road, enables the battery charging during the ordinary running of the vehicle. For this reason, it could be also a way to reduce the size of storage system and the total cost of the vehicle, of which about $30 \%$ is represented by the cost of battery. Literature shows different wireless charging systems, characterized by different coil topologies, different converters and numerous control strategies used for different purposes [6-9]. Of course, the main purpose is the efficiency maximization [10-11].

For this reason, it is useful to have as accurate as possible models for a careful system design. The core of wireless transmission systems is the inductive coupling between the coils, for which many of the models are based on magnetostatic and magnetodynamic analyzes [12]. This kind of models often use finite element analysis, which even if it is extremely accurate and a valid method able to well characterize the behavior of coils, the requirement of computational efforts is high. For this reason, in this paper a simplified mathematical model is presented and experimentally validated. Besides it also highlights how the use of a double receiver in a dynamic wireless charging system can reduce the cost of the entire charging infrastructure.

\section{WIRELESS TRANSFER SYSTEM DESIGN}

\section{A. Inductive Coupling}

The core of the IPT (Inductive Power Transfer) systems is the inductive coupling between the coils. Among the many typologies of antennas, Double-D (DD) coils are preferred for their reduced edge effects and for the high coupling effects for the same length of the material used if compared to other types such as planar circular ones [13].

The presented model is based on the principle of superposition for which the entire coil can be seen as a set of linear conductors. The geometrical construction of both transmitter and receiver coils is carried out, in Matlab environment, through the implementation of a matrix with dimensions $(\mathrm{N}+1 \times 3)$, whose elements represent the extreme space coordinates of the $\mathrm{N}$ linear conductors composing the antenna, linked by a continuity constrain. In fig. 1 , the geometric structure of two coupled DD coils is shown.

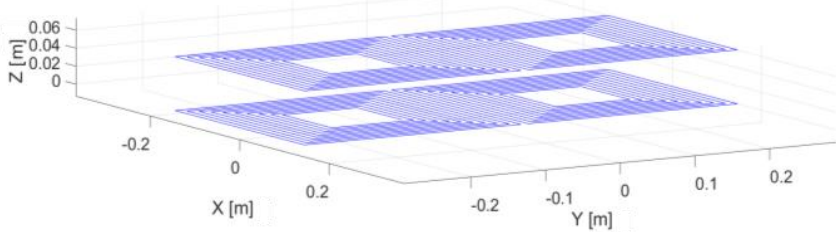

Fig. 1: Geometric structure of the coupled DD coils 


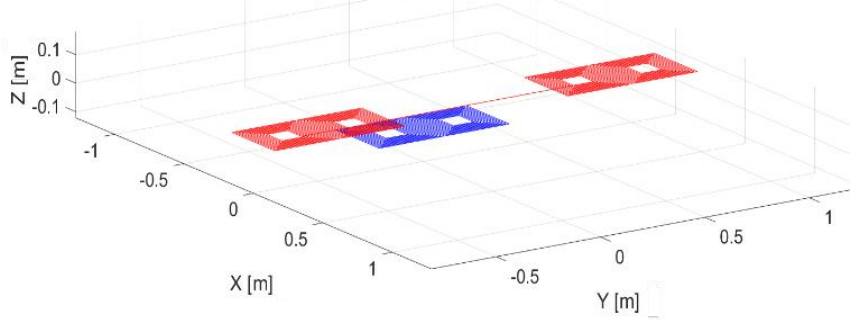

Fig. 2: Double receiver coil structure

In tab. 1 the geometric characteristics of the coils are reported. For the double receiver case, the receiver coil is composed by the series of two identical 10 turns DD coils having geometric characteristics equal to the transmitter one and distant each other $80 \mathrm{~cm}$, how shown in fig. 2 .

TABLE I. COILS GEOMETRIC CHARACTERISTICS

\begin{tabular}{|l|c|c|c|}
\hline & Length & Dimensions & Wire Section \\
\hline $\begin{array}{l}\text { Transmitter/single } \\
\text { receiver }\end{array}$ & $15,58 \mathrm{~m}$ & $58 \mathrm{~cm} \times 29 \mathrm{~cm}$ & $2,5 \mathrm{~mm}^{2}$ \\
\hline Double receiver & $32,96 \mathrm{~m}$ & $196 \mathrm{~cm} \times 29 \mathrm{~cm}$ & $2,5 \mathrm{~mm}^{2}$ \\
\hline
\end{tabular}

Thanks to the discretization of the DD coil in a finite number of conductors, it is possible to evaluate each single contribute of the potential vector and then calculate the selfinductance and mutual inductance values through the principle of superposition.

Flux $\Phi$ associate to the system can be valued as follow:

$\Phi(B)=\int_{S} \vec{B} \cdot d S=\mu \int_{S} \vec{H} \cdot d S$

in which $B$ is the magnetic induction, $H$ is the magnetic field, $\mu$ is the magnetic permeability and $\mathrm{S}$ the integrating surface, then applying the Stocks, (1) can be reformulated in:

$\mu \int_{S} \vec{H} \cdot d S=\mu \int_{S} \operatorname{rot} \vec{V} \cdot d S=\mu \int_{l} \vec{V} \cdot d \bar{l}$

in which $l$ is the surface contour and $V$ being the potential vector. Each element composing the coil, having length $L$, radius $r$ and carrying the current $I$ can be considered independently, and then it is possible to calculate the potential vector in a point $P(x, y)$ as follow:

$$
V(P)=\frac{I}{4 \pi}\left(\operatorname{Arcsinh} \frac{x+\frac{L}{2}}{y}-\operatorname{Arcsinh} \frac{x-\frac{L}{2}}{y}\right)
$$

This expression must be integrated along each conductor. Naturally, only those ones for which the scalar product between the generated potential and the induced element side path is not equal to zero can interact each other.

The potential vector is referred to the midpoint of the induced $i$-th element and it is valued on the surface in order to not involve the current distribution and then the internal potential vector. It can be expressed as reported in (4) and (5). Naturally, for the evaluation of the self-potential, the exact formula (4) can be applied while the calculation of the potential induced by the $j$-th element on the $i$-th element is approximated, as shown in (5), in order to simplify the algorithm, by taking into account $V_{j}\left(i_{m}\right)$ which is the potential on the midpoint of the $i$-th element and $\Delta l_{i j}=l_{i} \cdot \cos (\alpha)$ that is the $i$-th element projection on the $j$-th. The angle $\alpha$ is determined through the difference between $\alpha_{i}$ and $\alpha_{j}$, formed by the $i$-th element and the $j$-th element with a common direction.

$i V i i=\frac{I}{4 \pi}\left(2 L A r c \sinh \frac{L}{r}-2 \sqrt{L^{2}+r^{2}}+2 r\right)$

$i V_{j i}=\int_{-\frac{L_{i}}{2}}^{\frac{L_{i}}{2}} V_{j} \cdot d l_{i} \cong V_{j}\left(i_{m}\right) \Delta l_{i j}$

Then, the self-inductance value can be calculated as in (6) by implementing a matrix $i V$ having dimension $(i \times j)$, which rows elements represent the contributes of the potential vectors acting on each linear conductor.

$L=\frac{\mu}{I} \sum_{i, j=1}^{N} i V_{i, j}$

The value of the calculated self-inductance for the single DD coil is equal to $5,68 \times 10^{-5} \mathrm{H}$ while for the series DD coils employed as double receiver it is equal to $11,26 \times 10^{-5} \mathrm{H}$.

By following the same principle, the mutual-inductance value is calculated considering each potential contribute of the $i$-th elements of the transmitter coil on the $j$-th elements of the receiver coil. In order to simulate the sliding of the receiver antenna on the transmitter one, a for cycle is implemented varying the Y-misalignment between the coils. The trends of the mutual inductance for both single receiver and double receiver cases are shown in fig.3.

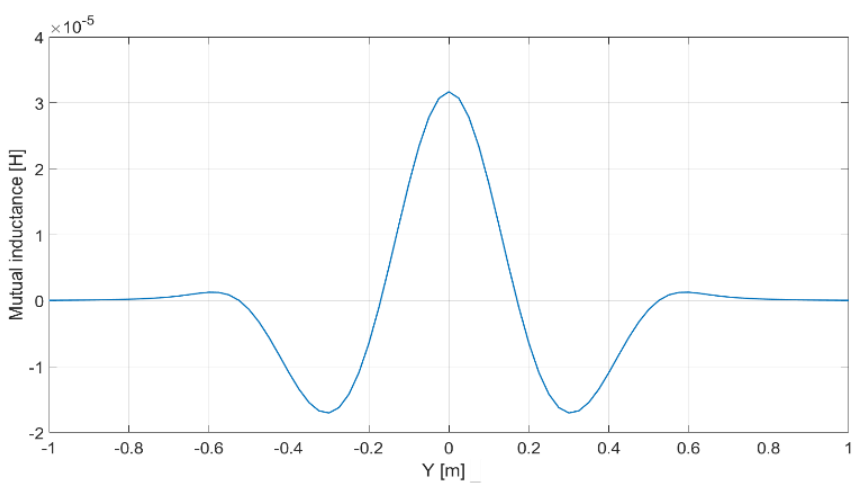

(a)

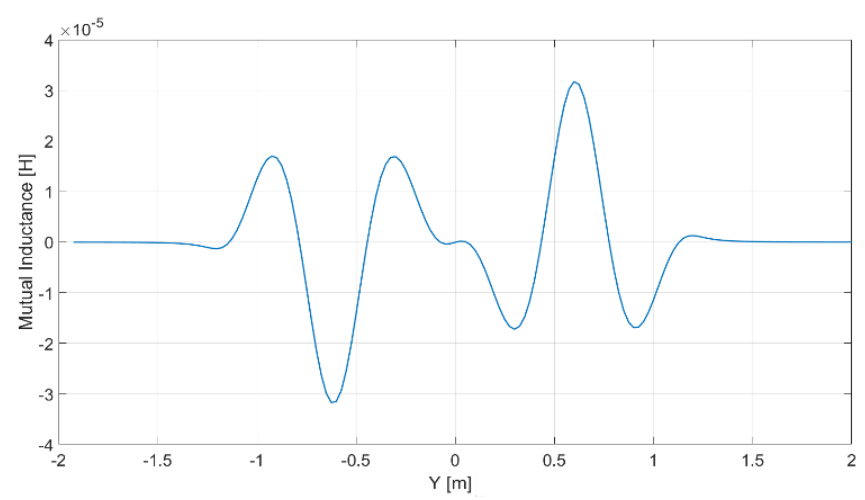

(b)

Fig. 3: Mutual inductance trend for single receiver (a) and double receiver (b) 


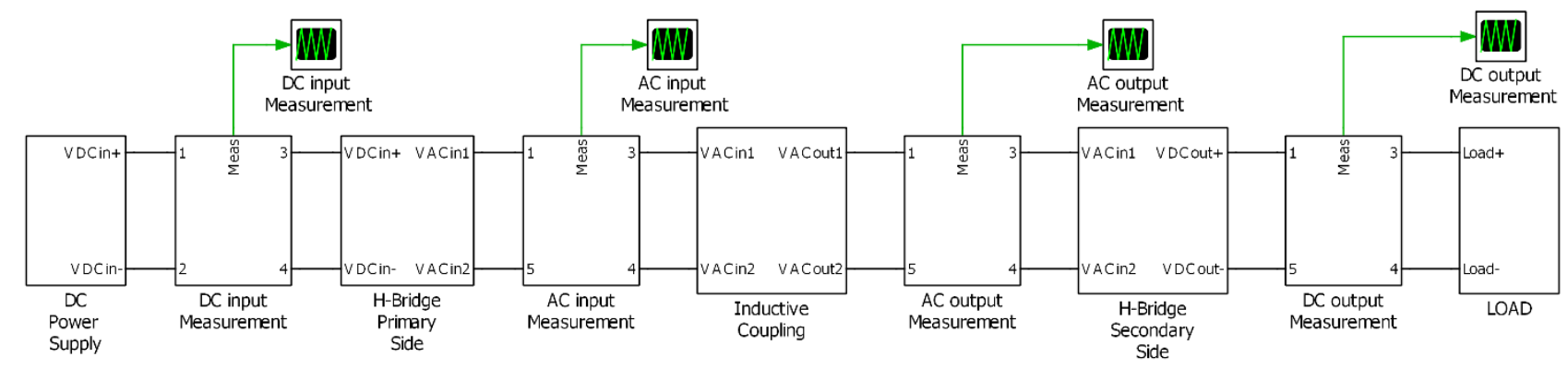

Fig. 4: Block scheme of the dynamic wireless charging system

\section{B. Compensation Networks and Power Converters}

In order to maximize the power transfer, a compensation network is needed on both primary and secondary side and a Series-Compensation is chosen in order to avoid the dependence of the resonant frequency with the mutual inductance which is variable. By choosing a resonant working frequency equal to $100 \mathrm{kHz}$, the compensation network is equal to $4,6 \times 10^{-8} \mathrm{~F}$ for a single antenna.

At the primary side, by starting from a DC source, the alternate current at the resonant frequency is delivered to the dynamic inductive coupling equipped by the compensation network, previously described, thanks to an H-Bridge converter. At the secondary side, another H-bridge converter, employed as a diode rectifier, is interposed between the receiver system and the load, in this case equal to a $10 \Omega$ resistance. The block scheme, shown in fig. 4, is implemented in PLECS environment. Simulations results will be shown in paragraph IV, compared with the experimental results.

\section{SYSTEM REALIZATION AND EXPERIMENTL VALIDATION}

\section{A. System components}

The simulated DD coils are realized manually in copper wire (fig. 5) and the self-inductance value is measured thanks to an RLC Meter. It is equal to $5,7 \times 10^{-5} \mathrm{H}$, verifying the model obtained value. The compensation networks are realized by the employment of a PET capacitors bench as reported in fig. 6 .

The proposed H-Bridge converters are built in laboratory. The IRFB4410 power N-Channel MOSFETs [14] have been chosen as switches. To drive the $4 \mathrm{~N}$-Channel MOSFETs of the converter, the HIP4081A driver [15] is used. For a proper operation, an external Bootstrap circuit is needed. For the experimental tests the primary side H-Bridge is controlled by the use of Arduino Due Shield, programmed to send two PWM signals phase-shifted of an angle equal to $90^{\circ}$ with a fixed duty cycle equal to $50 \%$ and frequency equal to the resonance one of the system. The secondary side converter is used as a diode rectifier. In fig. 6 a single converter is shown.

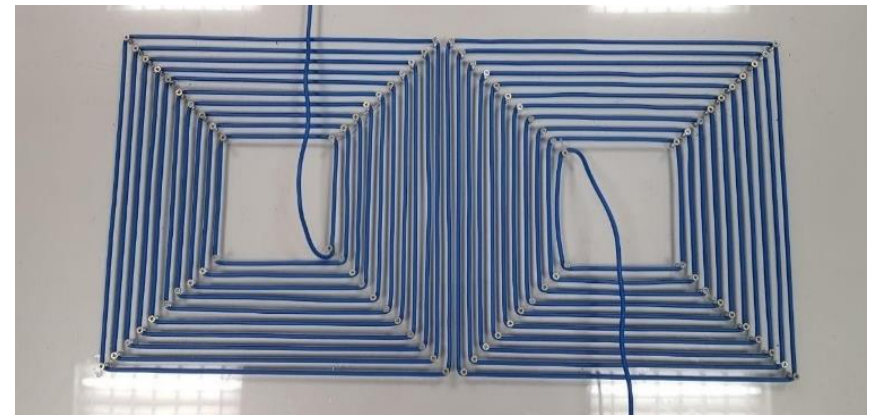

Fig. 5: DD coil realized

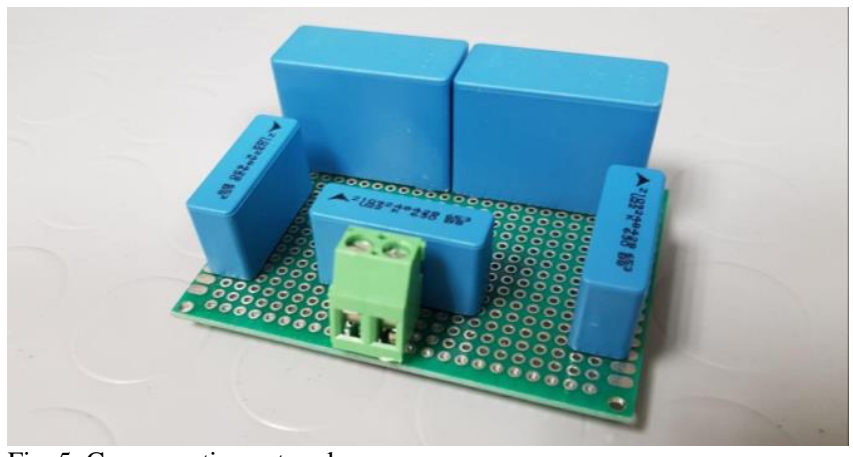

Fig. 5: Compensation network

\section{B. Experimental tests}

An aluminum track was made to constrain the passage of a trolley on which the secondary side of the system is mounted at a height of $6 \mathrm{~cm}$. The trolley is driven by a controlled prime motor to ensure a constant speed equal 0.1 $\mathrm{m} / \mathrm{s}$.

Three tests were carried out. The first static test was conducted with a single receiver in the absence of misalignment to verify correct system operation. Subsequently, at the previously indicated speed, two dynamic tests were curried out. The first one with a single receiver, the second one with a double receiver. The load in all cases is equal to $10 \Omega$.

In fig. 7 the test bench is shown. It consists on the dynamic wireless transfer system and on the following instrumentation:

- $\quad$ DC power supply 30V 10A;

- differential voltage probes;

- Current probes;

- $\quad$ Primary side oscilloscope;

- $\quad$ Secondary side oscilloscope;

- $\quad$ Electronic load 0-60V / 0-60A.

Then the input and output quantities have been acquired. The results are discussed in the next section.

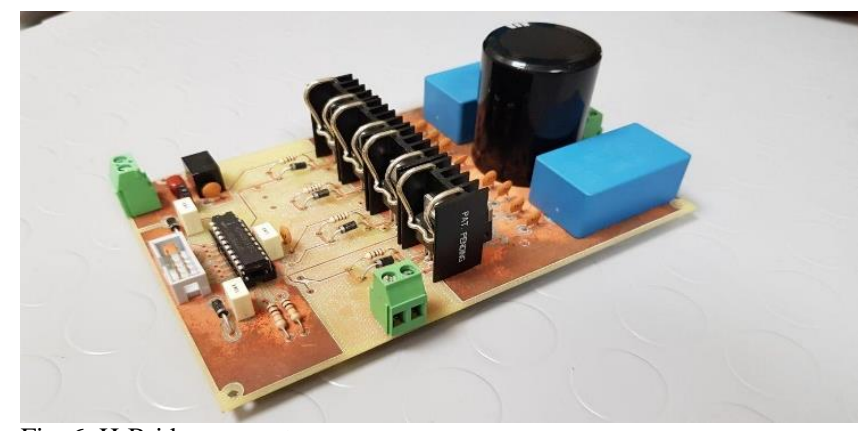

Fig. 6: H-Bridge converter 


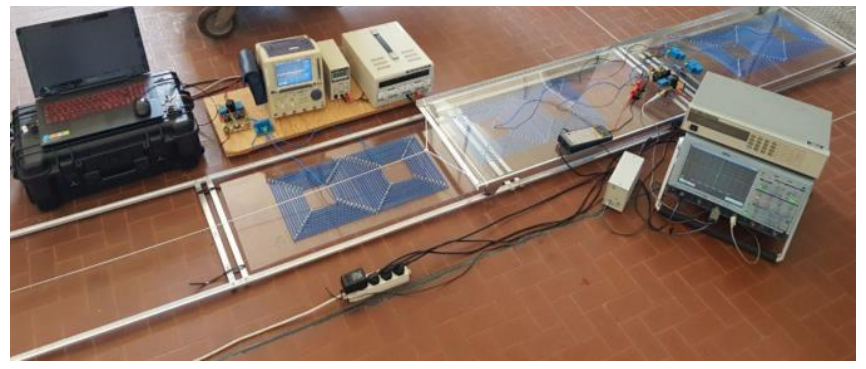

Fig. 7: Test bench setup

\section{RESULTS}

The first static test carried out shows how the model is close to the real behavior of the system. In fig. 8 (a) it is possible to notice in red the AC output current, in green the AC output voltage and in blue the load voltage, while in fig. 8 (b) the oscilloscope acquisition of the same quantities is shown. (5V/div for the AC signals, $3 \mathrm{~V} / \mathrm{div}$ for DC yellow signal).

In fig. 9 it is possible to notice the voltage and the current on the load in the case of a single receiver. In (a) the simulation results show in blue the voltage trend and in red the current and in (b) the oscilloscope acquisition (1s/div) shows in yellow the voltage trend and in red the current one. In both, the current is scaled up by a factor equal to 5 .

In fig. 10 the voltage and the current on the load for a double receiver are shown. In (a) the simulation results show in blue the voltage trend and in red the current and in (b) the oscilloscope acquisition ( $2 \mathrm{~s} / \mathrm{div}$ ) shows in yellow the voltage trend and in red the current one. In both, the current is scaled up by a factor equal to 5 .

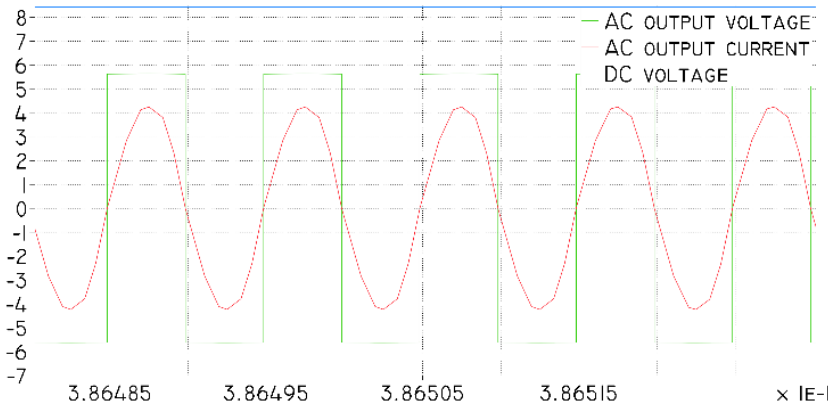

(a)

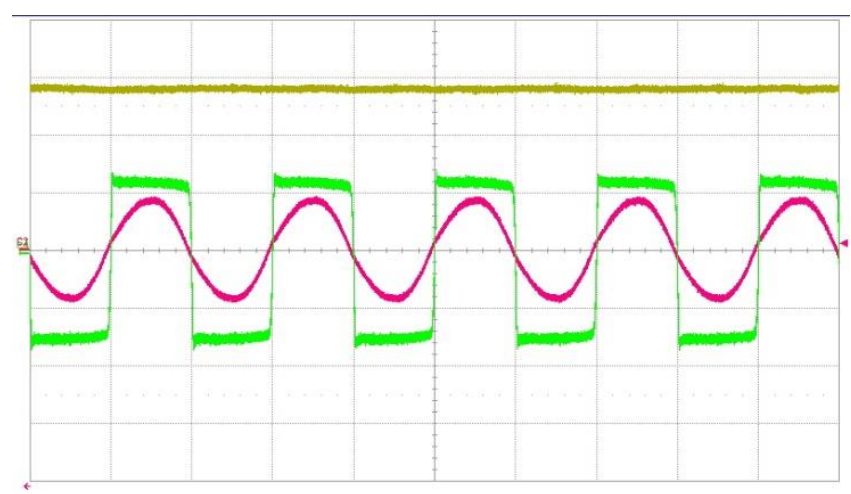

(b)

Fig. 8: Output quantities, simulation (a) and oscilloscope acquisition (b)

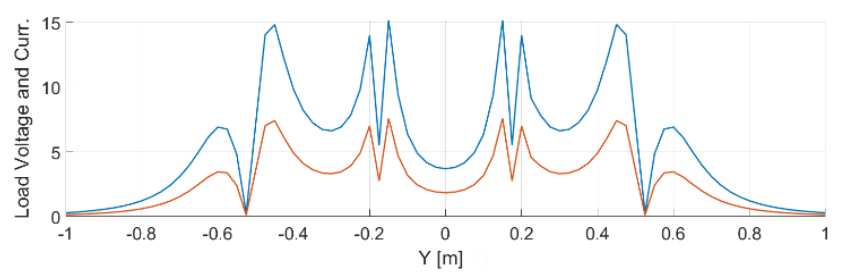

(a)

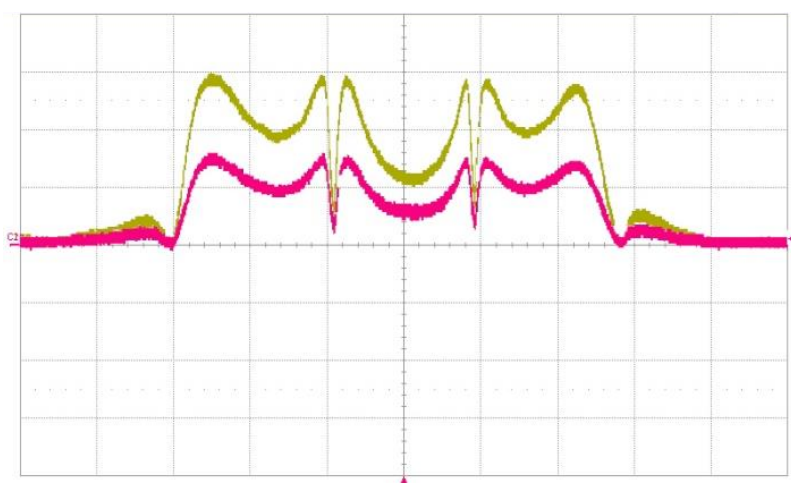

(b)

Fig. 9: Load voltage and current trends for single receiver, (a) simulation, (b) oscilloscope acquisition

It is possible to notice how the waveforms of voltage and current on the load with the presence of the double receiver formed by two DD coils identical to the transmitter and placed in series at the distance $d$ is equivalent to the waveforms that would occur if a single receiver swiped over two transmitters placed at distance $d$.

This means that by the use of a double receiver configuration whose distance between the coils is equal to $d$, it is possible to replicate the same waveforms by placing transmitters with a distance greater than $d$.

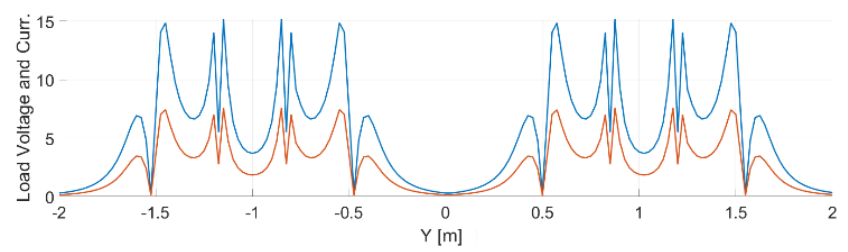

(a)

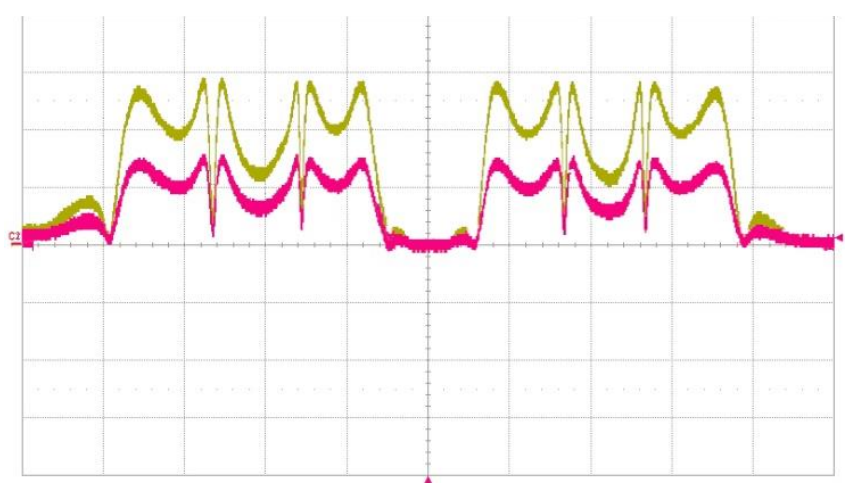

(b)

Fig. 9: Load voltage and current trends for double receiver, (a) simulation, (b) oscilloscope acquisition 
In this way, thanks to the reduction of the number of transmitters per $\mathrm{km}$ of road, it is possible to reduce significantly the costs of the entire dynamic charging infrastructure.

\section{CONCLUSIONS}

In this paper a low power prototype of a dynamic double receiver IPT system for electric vehicle is presented. A simplified mathematical model for the DD coils design is described. It is based on the superposition principle which allows the coils to be discretized into a finite number of linear conductors to calculate the self-inductance and mutual inductance values by considering the vector potential induced by each element. This model has been experimentally validated and it has been shown that unlike complex finite element analyzes, this model lends itself well to a simplified design of coils.

The entire dynamic wireless charging system is studied in simulation and each part made in laboratory. Experimental tests were curried on showing how the model of the entire system created in simulation is close to real behavior. It was also shown how through the use of a double receiver it is possible to reduce the number of transmitters and therefore make significantly lower the costs of the charging infrastructure.

\section{ACKNOWLEDGMENT}

This work was financially supported by PON R\&I 20152020 "Propulsione e Sistemi Ibridi per velivoli ad ala fissa e rotante - PROSIB", CUP no:B66C18000290005, by H2020ECSEL-2017-1-IA-two-stage "first and european sic eightinches pilot line-REACTION", by Prin 2017Settore/Ambito di intervento: PE7 linea C - Advanced powertrains and -systems for full electric aircrafts, by PON R\&I 2014-2020 - AIM (Attraction and International Mobility), project AIM1851228-1 and by ARS01_00459-PRJ-0052 ADAS+ "Sviluppo di tecnologie e sistemi avanzati per la sicurezza dell'auto mediante piattaforme ADAS".

\section{REFERENCES}

[1] Z. Zhang, H. Pang, A. Georgiadis and C. Cecati, "Wireless Power Transfer-An Overview," in IEEE Transactions on Industrial Electronics, vol. 66, no. 2, pp. 1044-1058, Feb.

[2] F. Pellitteri, M. Caruso, V. Castiglia, A. O. Di Tommaso, R. Miceli and L. Schirone, "An inductive charger for automotive applications," IECON 2016 - 42nd Annual Conference of the IEEE Industrial Electronics Society, Florence, 2016, pp. 4482-4486.

[3] H. Chu, X. Qu, S. Wong and C. K. Tse, "Design of an IPT Battery Charger with Double-sided LCC Compensation," 2018 IEEE Energy Conversion Congress and Exposition (ECCE), Portland, OR, 2018, pp. 1184-1189.

[4] N. Campagna, V. Castiglia, R. Miceli and F. Pellitteri, "A Bidirectional IPT system for Electrical Bicycle Contactless Energy Transfer," 2019 8th International Conference on Renewable Energy Research and Applications (ICRERA), Brasov, Romania, 2019, pp. 1063-1068.

[5] A. Triviño-Cabrera, J. Aguado and J. M. González, "Analytical characterisation of magnetic field generated by ICPT wireless charger," in Electronics Letters, vol. 53, no. 13, pp. 871-873, 226 2017.

[6] G. R. Kalra, M. G. S. Pearce, S. Kim, D. J. Thrimawithana and G. A. Covic, "Measuring the Q-factor of IPT Magnetic Couplers," 2019 IEEE PELS Workshop on Emerging Technologies: Wireless Power Transfer (WoW), London, United Kingdom, 2019, pp. 34-38.

[7] S. Kim, G. A. Covic and J. T. Boys, "Comparison of Tripolar and Circular Pads for IPT Charging Systems," in IEEE Transactions on Power Electronics, vol. 33, no. 7, pp. 6093-6103, July 2018.

[8] H. Hu, T. Cai, S. Duan, X. Zhang, J. Niu and H. Feng, "A Parallel Topology for Modularized IPT Systems," 2019 IEEE Energy Conversion Congress and Exposition (ECCE), Baltimore, MD, USA, 2019, pp. 1245-1250.

[9] Nutwong, A. Sangswang and S. Naetiladdanon, "Output voltage control of the SP topology IPT system using a primary side controller," 2016 13th International Conference on Electrical Engineering/Electronics, Computer, Telecommunications and Information Technology (ECTI-CON), Chiang Mai, 2016, pp. 1-5.

[10] Y. Chen, R. Mai, Y. Zhang, M. Li and Z. He, "Improving Misalignment Tolerance for IPT System Using a Third-Coil," in IEEE Transactions on Power Electronics, vol. 34, no. 4, pp. 3009-3013, April 2019.

[11] Y. Shou, B. Luo, S. Xiong, G. Zhu, J. Lu and J. V. Wang, "Efficiency Analysis in IPT System Based on LCC Compensation Network," 2019 10th International Conference on Power Electronics and ECCE Asia (ICPE 2019 - ECCE Asia), Busan, Korea (South), 2019, pp. 22582263.

[12] A. A. S. Mohamed, A. Berzoy and O. A. Mohammed, "Physics-based FE model and analytical verification of bi-directional inductive wireless power transfer system," 2016 IEEE/ACES International Conference on Wireless Information Technology and Systems (ICWITS) and Applied Computational Electromagnetics (ACES), Honolulu, HI, 2016, pp. 1-2

[13] X. CHEN, W. LU, Y. DONG and H. JIANG, "Magnetizer Optimization of DD Type Coils for EV Wireless Charging System," 2019 22nd International Conference on Electrical Machines and Systems (ICEMS), Harbin, China, 2019, pp. 1-4

[14] IFR4410 N-Channel MOSFET Datasheet, online available.

[15] HIP4081A driver Datasheet, online available. 